\title{
Synchronization of Uncertain Chaotic System with Unknown Response System Parameters
}

\author{
${ }^{1, \mathrm{a}}$ Zhanlei Shang, ${ }^{1, \mathrm{~b}}$ Wenjun Song, ${ }^{2, \mathrm{c}}$ Ruiqi Wang and ${ }^{3, \mathrm{~d}}$ Junwei Lei \\ ${ }^{1}$ College of Computer and Communication Engineering, Zhengzhou University of \\ Light Industry, Zhengzhou 450002, China \\ ${ }^{2}$ Department of Armament Engineering, Naval Aeronautical and Astronautical \\ University, Yantai Shandong 264001, China \\ ${ }^{3}$ Department of Control Engineering, Naval Aeronautical and Astronautical \\ University, Yantai Shandong 264001, China \\ ashangzl@163.com, ${ }^{b} 11395079 @ q q . c o m,{ }^{c}$ richkey1980@gmail.com, \\ deijunwei@126.com,
}

\begin{abstract}
Synchronization of chaotic system is meaningful and widely used in secure communication. A kind of robust adaptive synchronization method was proposed to solve a special chaotic synchronization problem that both response system and driven system have unknown parameters and uncertain nonlinear functions. And the difficulty is also caused by the different structures between master chaotic system and slave chaotic system. A kind of update law of estimation of unknown parameters were designed by choose a proper Lyapunov energy function. And the stability of the whole system is guaranteed by Lyapunov stability theorem. At last, detailed numerical simulation was done to show the rightness of the proposed method.
\end{abstract}

Keywords: chaotic system; synchronization; adaptive control; nonlinear function; the Lipscitz condition; robustness;

\section{Introduction}

Synchronization of chaotic system was studied by many researchers for its possible application in secure communication ${ }^{[1-23]}$. The synchronization of chaotic systems with different structures was researched by Jian Huang ${ }^{[1]}$. Considering the situation that the parameters of driven system and response system are totally unknown, synchronization between LS chaotic system and CYQY chaotic system, and synchronization between LS chaotic system and Chen chaotic system are realized respectively with adaptive method. But only unknown parameters are considered and the situation of chaotic system with uncertain nonlinear functions are not discussed in this paper.

The situation that the driven chaotic system has the same structure with the response system is discussed by Ju H. Park in paper ${ }^{[2,3]}$. A kind of feedback synchronization method based on Lyapunov function and LMI method are proposed for Genesio -Tesi chaotic systems with three unknown parameters. And the criterion for the existence of control law are solved by LMI method. So the disadvantage of this paper is that the above method is designed for the special Genesio -Tesi systems with the same structure. And the uncertainties of chaotic system are not fully discussed so it is not easy to be applied in a general chaotic system with both unknown parameters and other uncertainties.

Xianyong $\mathrm{Wu}^{[4]}$ discussed the synchronization problem of chaotic system with different structure but the response system does not contain any unknown parameter. And a kind of adaptive chaotic synchronous controller was designed for the synchronization between special Chen chaotic system and Henon-Heiles chaotic system. And the update law of estimation of unknown parameters are constructed based on Lyapunov stability 
theorem. But also the system uncertainties are assumed to be simple and complex situation of system uncertainties are not considered.

In this paper, synchronization problem of a kind of general chaotic systems was discussed and the response system has the same dimension but different structure with the driven system. Both the unknown parameters and uncertain nonlinear functions are considered in driven system and response system, and a kind of robust adaptive synchronous controller were designed based on Lyapunov stability theorem to fulfill the synchronization. At last, a four dimension chaotic system was taken as an example to do the numerical simulation and the simulation result testified the rightness and effectiveness of the proposed method[4-6].

\section{Problem Description}

Consider the below driven chaotic system and response chaotic system[7-10], the response system has unknown parameters and uncertain nonlinear functions, and the driven system with unknown parameters and nonlinear function can be described as follows:

The driven chaotic system model can be written as

$$
\stackrel{t}{*} f_{x}(x)+F_{x}(x) \theta_{x}+\Delta_{x}(x, t)
$$

The response chaotic system model can be written as

$$
k=f_{y}(y)+F_{y}(y) \theta_{y}+\Delta_{y}(y, t)+b u
$$

Taken a four dimension chaotic system as an example, the driven system can be expanded as

$$
\begin{array}{r}
=f_{x 1}\left(x_{1}, \mathrm{~L}, x_{4}\right)+\sum_{j=1}^{p_{1}} F_{x 1 j}\left(x_{1}, \mathrm{~L}, x_{4}\right) \theta_{x 1 j}+\sum_{j=1}^{p_{2}} \Delta_{x 1 j}(x, t) \\
\&=f_{x 2}\left(x_{1}, \mathrm{~L}, x_{4}\right)+\sum_{j=1}^{p_{1}} F_{x 2 j}\left(x_{1}, \mathrm{~L}, x_{4}\right) \theta_{x 2 j}+\sum_{j=1}^{p_{2}} \Delta_{x i j}(x, t) \\
=f_{x 3}\left(x_{1}, \mathrm{~L}, x_{4}\right)+\sum_{j=1}^{p_{1}} F_{x 3 j}\left(x_{1}, \mathrm{~L}, x_{4}\right) \theta_{x 3 j}+\sum_{j=1}^{p_{2}} \Delta_{x 2 j}(x, t) \\
=f_{x 4}\left(x_{1}, \mathrm{~L}, x_{4}\right)+\sum_{j=1}^{p_{1}} F_{x 4 j}\left(x_{1}, \mathrm{~L}, x_{4}\right) \theta_{x 4 j}+\sum_{j=1}^{p_{2}} \Delta_{x 4 j}(x, t)
\end{array}
$$

And the slave response system can be expanded as

$$
\begin{aligned}
& \&=f_{y 1}\left(y_{1}, \mathrm{~L}, y_{4}\right)+\sum_{j=1}^{p_{3}} F_{y 1 j}\left(y_{1}, \mathrm{~L}, y_{4}\right) \theta_{y 1 j}+\sum_{j=1}^{p_{4}} \Delta_{y 1 j}(y, t)+b_{1} u_{1} \\
& =f_{y 2}\left(y_{1}, \mathrm{~L}, y_{4}\right)+\sum_{j=1}^{p_{3}} F_{y 2 j}\left(y_{1}, \mathrm{~L}, y_{4}\right) \theta_{y 2 j}+\sum_{j=1}^{p_{4}} \Delta_{y 2 j}(y, t)+b_{2} u_{2} \\
& \&=f_{y 3}\left(y_{1}, \mathrm{~L}, y_{4}\right)+\sum_{j=1}^{p_{3}} F_{y 3 j}\left(y_{1}, \mathrm{~L}, y_{4}\right) \theta_{y 1 j}+\sum_{j=1}^{p_{4}} \Delta_{y 3 j}(y, t)+b_{3} u_{3} \\
& =f_{y 4}\left(y_{1}, \mathrm{~L}, y_{4}\right)+\sum_{j=1}^{p_{3}} F_{y 4 j}\left(y_{1}, \mathrm{~L}, y_{4}\right) \theta_{y 4 j}+\sum_{j=1}^{p_{4}} \Delta_{y 4 j}(y, t)+b_{3} u_{4}
\end{aligned}
$$

where $\theta_{x}$ and $\theta_{y}$ are unknown parameter and $\Delta_{x}$ is unknown nonlinear function, so the number of unknown parameters is $n^{*}\left(p_{1}+p_{3}\right)$, and the number of nonlinear function is $n *\left(p_{2}+p_{4}\right)$.

For the situation that both driven system and response system have unknown parameters and uncertain nonlinear functions, the objective of synchronization problem of chaotic system is to design a control law $u=u\left(x, y, \hat{\theta}_{x}, \hat{q}_{x}, \hat{\theta}_{y}, \hat{q}_{y}\right), \theta_{x}=f\left(x, y, \hat{\theta}_{x}\right)$, 
$\mathscr{q}_{x}^{\&}=f\left(x, y, \hat{q}_{x}\right), \underset{\theta}{\&}=f\left(x, y, \hat{\theta}_{y}\right), \stackrel{\&}{\&}=f\left(x, y, \hat{q}_{y}\right)$ such that the state of response system can track the state of driven system, then it means $y \rightarrow x$.

\section{Assumption}

Assumption 1: The response system has the same structure of the driven system and they have the same dimension $f_{x i}=f_{y i}$.

Assumption 2: Some parts of driven system are known, it means $F_{x i j}$ and $f_{x i}$ are known.

Assumption 3: The response system is totally known, it means that $f_{y i}$ and $b_{i}$ are known $^{[11-14]}$.

Assumption 4: The nonlinear function of driven system satisfies following conditions. It means : for $1 \leq i \leq n, 1 \leq j \leq p_{2}$, there exists a unknown constant $q_{i j}^{*} \leq d_{i j}$ such that

$$
\left|\Delta_{x i j}(X, t)\right| \leq q_{i j}^{*} \psi_{i j}(X)
$$

where $d_{i j}$ is a known constant and $\psi_{i j}(X)$ is a known positive smooth function.

Assumption 5: The nonlinear function of response system satisfies following conditions. It means : for $1 \leq i \leq n, 1 \leq j \leq p_{4}$, there exists a unknown constant $q_{y i j}^{*} \leq d_{y i j}$ such that

$$
\left|\Delta_{y i j}(X, t)\right| \leq q_{y j}^{*} \psi_{y i j}(X)
$$

where $d_{y i j}$ is a known constant and $\psi_{y i j}(X)$ is a known positive smooth function ${ }^{[15-17]}$.

\section{The Design of Robust Adaptive Synchronization Controller}

Define an error variable as $z_{i}=y_{i}-x_{i}$. The error response system of the above driveresponse system can be written as

$$
\begin{aligned}
& \underset{\imath}{\&}=f_{y i}\left(y_{1}, \mathrm{~L}, y_{4}\right)-f_{x i}\left(x_{1}, \mathrm{~L}, x_{4}\right)+\sum_{j=1}^{p_{3}} F_{y i j}\left(y_{1}, \mathrm{~L}, y_{4}\right) \theta_{y i j}+\sum_{j=1}^{p_{4}} \Delta_{y i j}(y, t) \\
& -\sum_{j=1}^{p_{1}} F_{x i j}\left(x_{1}, \mathrm{~L}, x_{4}\right) \theta_{x i j}-\sum_{j=1}^{p_{2}} \Delta_{x i j}(x, t)+b_{i} u_{i}
\end{aligned}
$$

Design control law as follows

$$
\begin{aligned}
& u_{i}=f_{2 i}(x)\left[-f_{y i}\left(y_{1}, \mathrm{~L}, y_{4}\right)+f_{x i}\left(x_{1}, \mathrm{~L}, x_{4}\right)+\sum_{j=1}^{p_{1}} F_{x i j}\left(x_{1}, \mathrm{~L}, x_{4}\right) \hat{\theta}_{x i j}\right. \\
& \left.+\sum_{j=1}^{p_{2}} \hat{q}_{x i j} \psi_{x i j}(x)-\sum_{j=1}^{p_{3}} F_{y i j}\left(y_{1}, \mathrm{~L}, y_{4}\right) \hat{\theta}_{y i j}-\sum_{j=1}^{p_{2}} \hat{q}_{y i j} \psi_{y i j}(y)-f_{z i}\left(z_{i}\right)\right]
\end{aligned}
$$

Where

$$
\begin{gathered}
f_{2 i}(x)=b_{i}^{-1} \\
f_{z i}\left(z_{i}\right)=k_{i 1} z_{i}+k_{i 2} \frac{z_{i}}{\left|z_{i}\right|+\varepsilon_{i 1}}+k_{i 3} \frac{3}{2} z_{i}^{1 / 3} \exp \left(z_{i}^{2 / 3}\right)+k_{i 4} \operatorname{sign}\left(z_{i}\right)
\end{gathered}
$$

Then 


$$
\begin{aligned}
& z_{i} \&=z_{i}\left[-f_{z i}\left(z_{i}\right)-\sum_{j=1}^{p_{1}} F_{x i j}\left(x_{1}, \mathrm{~L}, x_{4}\right) \theta_{x i j}^{o}+\sum_{j=1}^{p_{2}}\left\{\hat{q}_{x i j} \psi_{i j}(x)-\Delta_{x i j}(x, t)\right\}\right. \\
& \left.+\sum_{j=1}^{p_{3}} F_{y i j}\left(y_{1}, \mathrm{~L}, y_{4}\right) \theta_{y i j}^{o}+\sum_{j=1}^{p_{4}}\left\{\Delta_{y i j}(y, t)-\hat{q}_{y i j} \psi_{y i j}(x)\right\}\right]
\end{aligned}
$$

Where

Considering that

$$
\theta_{x i j}^{o}=\theta_{x i j}-\hat{\theta}_{x i j}, \quad \theta_{y i j}^{o}=\theta_{y i j}-\hat{\theta}_{y i j}
$$

$$
\begin{aligned}
& z_{i} \sum_{j=1}^{p_{2}}\left\{\hat{q}_{x i j} \psi_{i j}(x)-\Delta_{x i j}(x, t)\right\} \leq \sum_{j=1}^{p_{2}}\left\{z_{i} \hat{q}_{x i j} \psi_{x i j}(x)+q_{x i j}{ }^{*}\left|z_{i}\right| \psi_{x i j}(x)\right\} \\
&= \sum_{j=1}^{p_{2}}\left\{\left|z_{i}\right| \psi_{x i j}(x)\left[\operatorname{sign}\left(z_{i}\right) \hat{q}_{x i j}+q_{x i j}{ }^{*}\right]\right\} \\
& z_{i} \sum_{j=1}^{p_{4}}\left\{\Delta_{y i j}(y, t)-\hat{q}_{y i j} \psi_{y i j}(x)\right\} \leq \sum_{j=1}^{p_{4}}\left\{-z_{i} \hat{q}_{y i j} \psi_{y i j}(y)+q_{y i j}{ }^{*}\left|z_{i}\right| \psi_{y i j}(y)\right\} \\
&=\sum_{j=1}^{p_{4}}\left|z_{i}\right| \psi_{y i j}(y)\left\{-\operatorname{sign}\left(z_{i}\right) \hat{q}_{y i j}+q_{y i j}{ }^{*}\right\}
\end{aligned}
$$

Define

Then

$$
\begin{aligned}
& q_{x i j}=q_{x i j}{ }^{*}+\operatorname{sign}\left(z_{i}\right) \hat{q}_{x i j} \\
& q_{y i j}=q_{y i j}{ }^{*}-\operatorname{sign}\left(z_{i}\right) \hat{q}_{y i j}
\end{aligned}
$$

$$
\begin{aligned}
& z_{i} \sum_{j=1}^{p_{2}}\left\{\hat{q}_{x i j} \psi_{x i j}(x)-\Delta_{x i j}(x, t)\right\} \leq \sum_{j=1}^{p_{2}}\left\{\left|z_{i}\right| \psi_{x i j}(x) \mathscr{q _ { x i j }}\right\} \\
& z_{i} \sum_{j=1}^{p_{4}}\left\{\hat{q}_{y i j} \psi_{y i j}(y)-\Delta_{y i j}(y, t)\right\} \leq \sum_{j=1}^{p_{4}}\left\{\left|z_{i}\right| \psi_{y i j}(y) \mathscr{q _ { y i j }}\right\}
\end{aligned}
$$

Considering that

$$
\theta_{x i j}^{\&}=\theta_{x i j}^{\&}-\theta_{x i j}^{\&}=-\theta_{x i j}^{\&}
$$

Design the adaptive adjustment law as

$$
\theta_{x i j}^{\&}=-z_{i} F_{x i j}\left(x_{1}, \mathrm{~L}, x_{4}\right)
$$

In the same way, it holds

$$
\begin{gathered}
\&_{x i j}=\operatorname{sign}\left(z_{i}\right) \underset{q_{x i j}}{\&} \\
\mathscr{\&}_{y i j}=-\operatorname{sign}\left(z_{i}\right) \underset{y i j}{\&}
\end{gathered}
$$

And design the adaptive adjustment law as

$$
\stackrel{\&}{\underset{q}{*}}=-z_{i} \psi_{x i j}(x) \stackrel{\&}{\&} \underset{y i j}{\&}=z_{i} \psi_{y i j}(y)
$$

Select a Lyapunov function as

$$
V=\sum_{i=1}^{n} z_{i}^{2}+\sum_{i=1}^{n} \sum_{j=1}^{p_{1}} \frac{1}{2}\left(\theta_{x i j}^{o}\right)^{2}+\sum_{i=1}^{n} \sum_{j=1}^{p_{2}} \frac{1}{2}\left(\mathscr{q}_{i j}\right)^{2}
$$

Solve its derivation and it is esay to get:

$$
\mathbb{L} \leq \sum_{i=1}^{n}-z_{i} f_{z i}\left(z_{i}\right) \leq 0
$$


According to Lyapunov stability theory, the system is stable and then $z_{i} \rightarrow 0$, so the synchronization of chaotic system can be realized.

\section{Numerical Simulation}

Take a four dimension hyper-chaotic system as an experiment object, The model of drive chaotic system can be written as follows.

$$
\begin{gathered}
\&=a\left(x_{2}-x_{1}\right)+k_{l b} x_{4} \cos x_{2} \\
\&=b x_{1}-k x_{1} x_{3}+k_{l b}\left(1+\sin \left(x_{2} x_{3}\right)\right) x_{2} \\
\&=-c x_{3}+h x_{1}^{2}+k_{l b}\left(2-\cos \left(x_{1} x_{2} x_{3} x_{4}\right)\right) x_{1} \\
\&=-d x_{1}+k_{l b} x_{3}\left(3+\sin \left(x_{1} x_{3}\right)\right)
\end{gathered}
$$

where $a, b, c, d$ are unknown parameters. The assumption conditions that uncertain nonlinear functions satisfy are the same with above. The parameters of response system are known. Its structure is shown as below ${ }^{[18-20]}$.

$$
\begin{gathered}
\mathscr{A}=a_{y}\left(y_{2}-y_{1}\right)+k_{l b}\left(1+\sin \left(y_{2} y_{3}\right)\right) y_{2}+u_{1} \\
\&=b_{y} y_{1}-k y_{1} y_{3}+k_{l b} y_{4} \cos y_{2}+u_{2} \\
\&=-c_{y} y_{3}+h y_{1}^{2}+k_{l b} y_{3}\left(3+\sin \left(y_{1} y_{3}\right)\right)+u_{3} \\
\&=-d_{y} y_{1}+k_{l b}\left(2-\cos \left(y_{1} y_{2} y_{3} y_{4}\right)\right) y_{1}+u_{4}
\end{gathered}
$$

Unknown parameters in the program are set as $\left(a_{y}, b_{y}, c_{y}, d_{y}\right)=(9,39,2.4,-10.5)$. The initial states of drive system are set as $\left(x_{1}, x_{2}, x_{3}, x_{4}\right)=(1,-1,2,-2)$. And the initial states of response system are set as $\left(y_{1}, y_{2}, y_{3}, y_{4}\right)=(-3,3,-5,5)$. The error system is shown as below.

$$
\begin{gathered}
\&=a_{y}\left(y_{2}-y_{1}\right)-a\left(x_{2}-x_{1}\right)-k_{l b} x_{4} \cos x_{2}+u_{1} \\
\&=b_{y} y_{1}-k_{1} y_{1} y_{3}-\left\{b x_{1}-k_{1} x_{1} x_{3}+k_{l b}\left(1+\sin \left(x_{2} x_{3}\right)\right) x_{2}\right\}+u_{2} \\
\&=-c_{y} y_{3}+h y_{1}^{2}-\left\{-c x_{3}+h x_{1}^{2}+k_{l b}\left(2-\cos \left(x_{1} x_{2} x_{3} x_{4}\right)\right) x_{1}\right\}+u_{3} \\
\&=-d_{y} y_{1}-\left\{-d x_{1}+k_{l b} x_{3}\left(3+\sin \left(x_{1} x_{3}\right)\right)\right\}+u_{4}
\end{gathered}
$$

The designed control law is as follows.

$$
\begin{aligned}
& u_{i}=f_{2 i}(x)\left[-f_{y i}\left(y_{1}, \mathrm{~L}, y_{4}\right)+f_{x i}\left(x_{1}, \mathrm{~L}, x_{4}\right)\right. \\
& +\sum_{j=1}^{p_{1}} F_{x i j}\left(x_{1}, \mathrm{~L}, x_{4}\right) \hat{\theta}_{x i j}+\sum_{j=1}^{p_{2}} \hat{q}_{x i j} \psi_{x i j}(x) \\
& \left.-\sum_{j=1}^{p_{3}} F_{y i j}\left(y_{1}, \mathrm{~L}, y_{4}\right) \hat{\theta}_{y i j}-\sum_{j=1}^{p_{2}} \hat{q}_{y i j} \psi_{y i j}(y)-f_{z i}\left(z_{i}\right)\right]
\end{aligned}
$$

Definition and selection of controller parameters are shown above. The simulation results are shown in following figures ${ }^{[21-23]}$. 


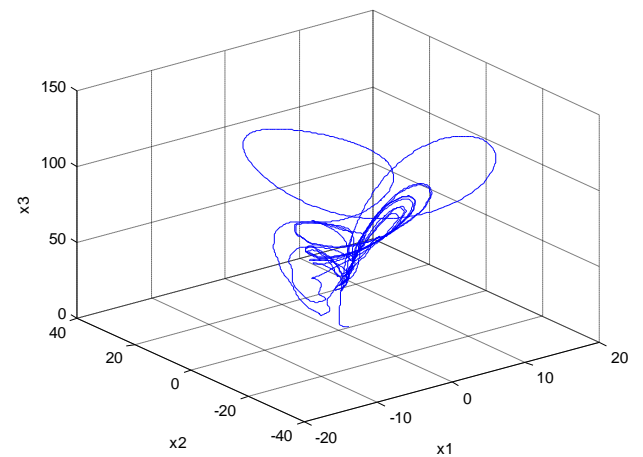

Figure 1. Trajectory of Uncontrolled Chaotic Sysmtes (1)

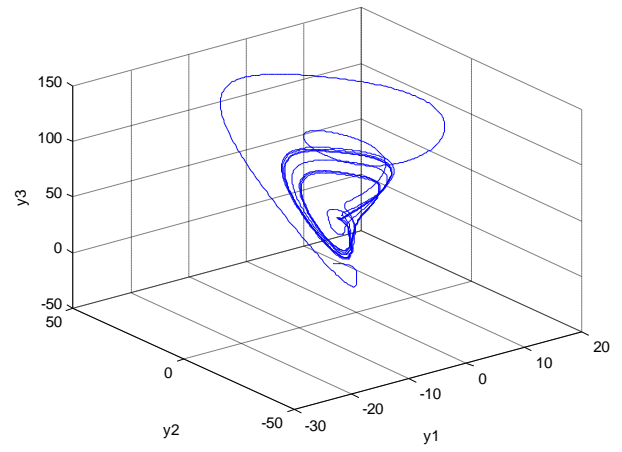

Figure 3. Trajectory of Uncontrolled Figure 4. Trajectory of Uncontrolled Chaotic Sysmtes (3)

Freedom movement trajectory of drive system and response system without control are shown by figure 1 to figure 4 . Comparison of two chaotic trajectory between drive system and response system without control are shown from figure 5 to figure 8 . It can be found from the diagram that two systems can not synchronize with each other.
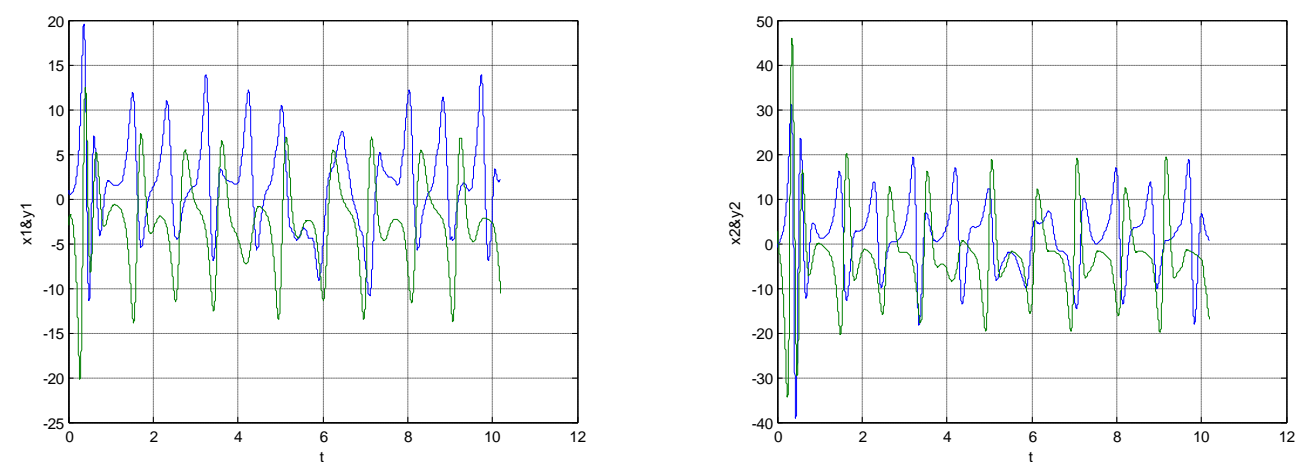

Fig.5 Trajectory of State x1 and y1 Figure 6. Trajectory of State x2 and y2 

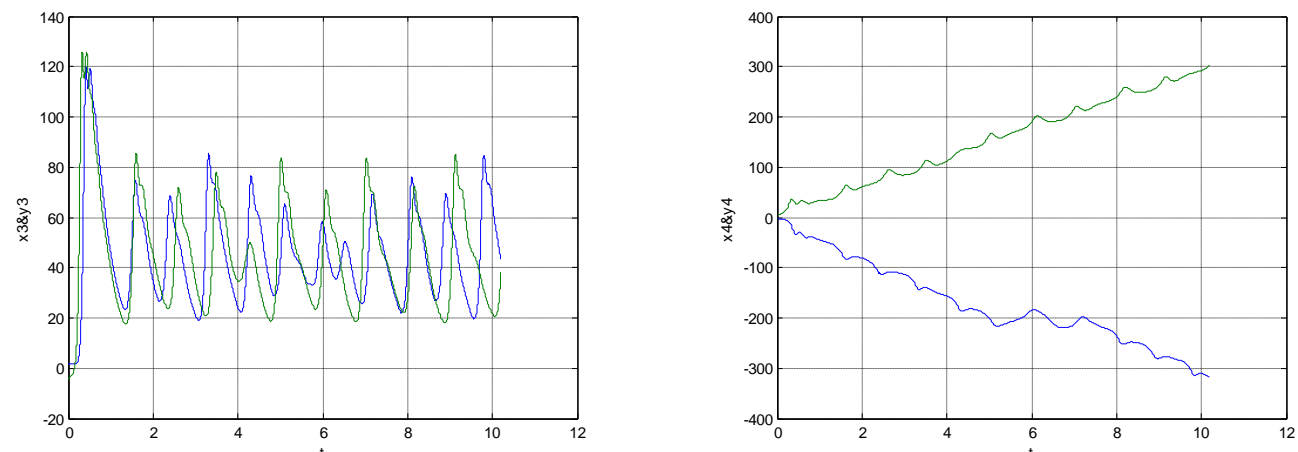

Fig.7 Trajectory of states $x 3$ and y3 Figure 8 . Trajectory of States $x 4$ and y4

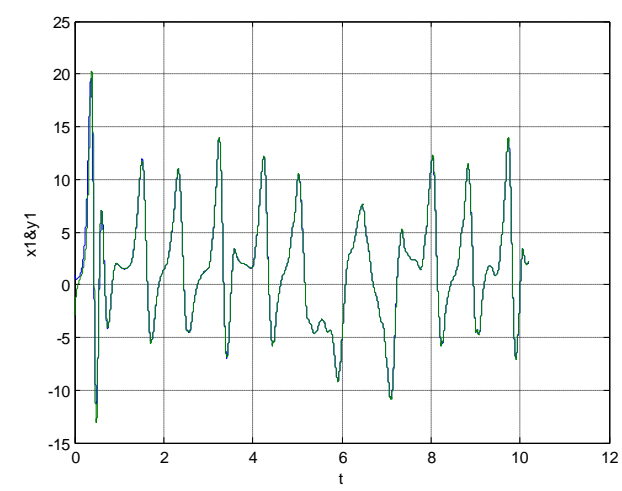

Figure 9. Tracing Curve of State $x 1$

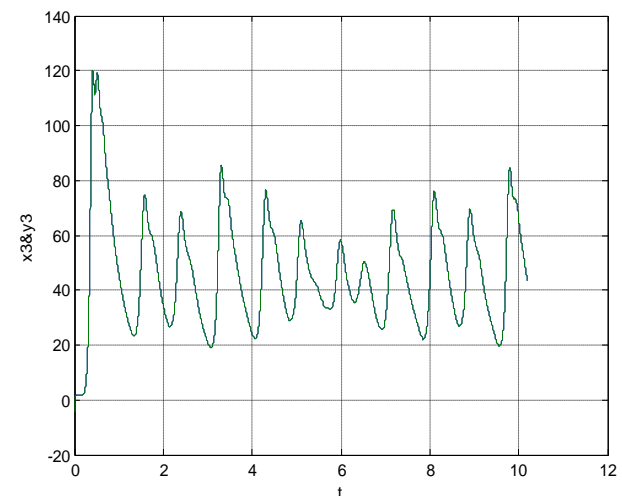

Figure 11. Tracing Curve of State $\times 3$

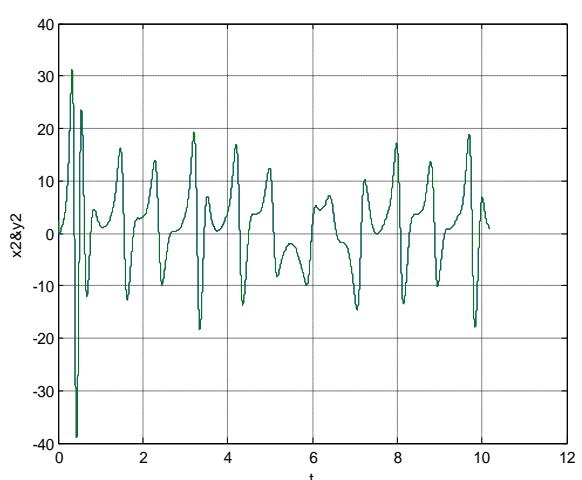

Figure 10. Tracing Curve of State x2

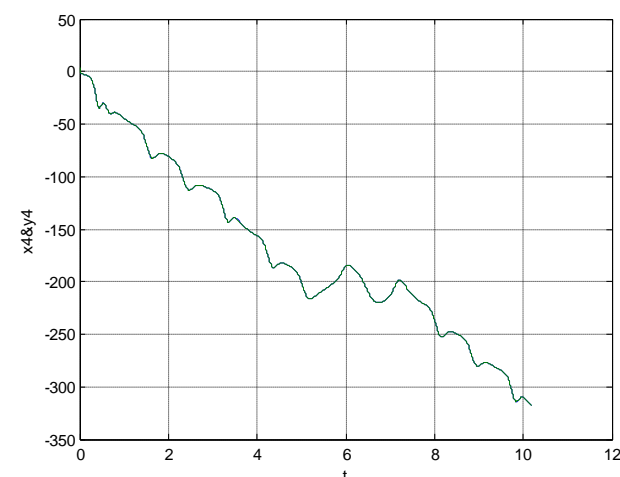

Figure 12. Tracing Curve of State $x 4$

It can be shown from figure 9 to figure 12 that response system can track drive system in the effect of synchronous controller. 


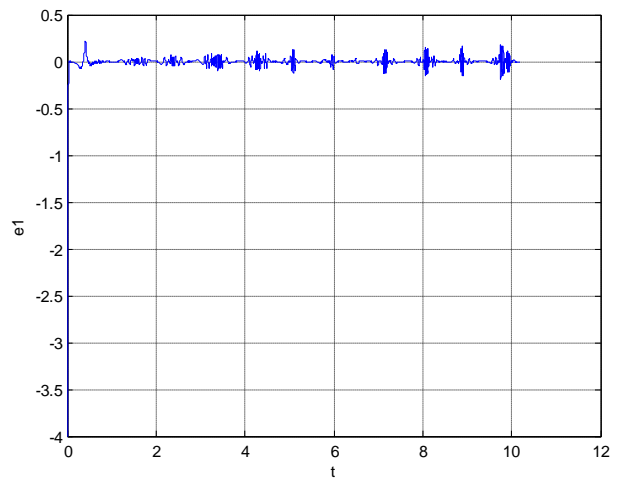

Figure 13. Error Curve of e1

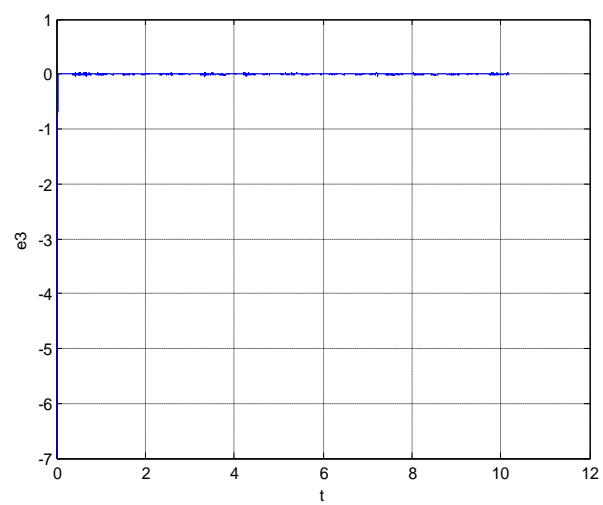

Figure 15. Error Curve of e3

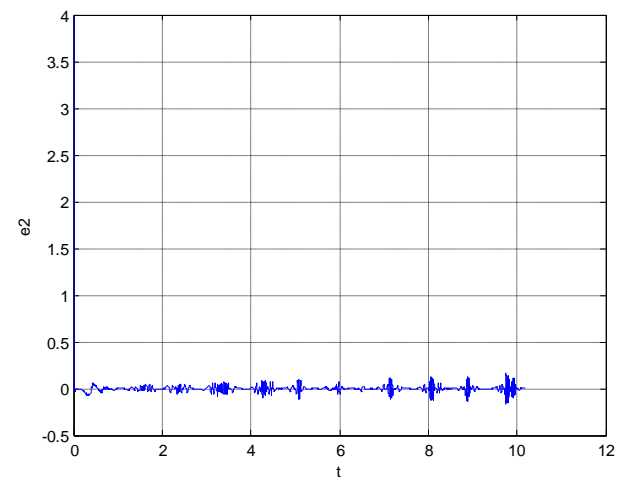

Figure 14. Error Curve of e2

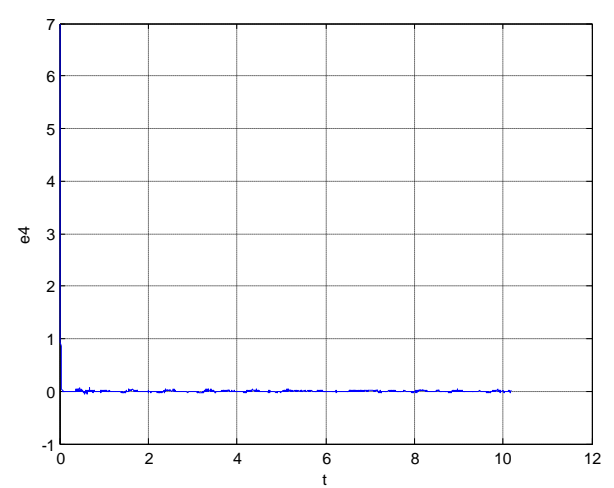

Figure 16. Error Curve of e4

In summary, the drive system and response system can achieve fast synchronization by using method in this paper in the drive systems with unknown parameters and uncertain nonlinear functions. In the same time, it can be shown from figure 13 to figure 16 that synchronization error can be stable in a small area near zero.

\section{Conclusions}

The synchronization problem of chaotic systems with unknown parameters and uncertain nonlinear functions in driven and response system was discussed. And the difficulty is also caused by the different structures between master chaotic system and slave chaotic system. Adaptive law was designed to cope with the unknown parameters and update law was designed to estimate unknown parameters. Also the robust strategy was adopted to treat the nonlinear functions. And the stability of the whole system is guaranteed by Lyapunov stability theorem. At last, numerical simulation of four dimension chaotic system was done to testify the rightness of the proposed method. So this method is proved to be effective in theory but in real engineering application, main difficulty will face is the nonlinear function introduced by the controller. It will lead the selection of simulation step to be difficult or the simulation should better be done with variable step simulation method or the system will be unstable. This is the main disadvantage caused by the introduction of terminal sliding mode items. But it also has obvious advantage that it the simulation step is set properly, the synchronization error will be small and the synchronization speed will be very quick. So it means that if those nonlinear control items are used in the control strategy, the simulation program and the realization of those algorithm should be done more carefully and exquisitely. Also, a 
more complex and more advance simulation program and simulation method is needed in order to realize those more complex nonlinear control items.

\section{Acknowledgment}

The author wish to thank his friend Heidi in Angels (a town of Canada) for her help , and thank his classmate Amado in for his many helpful suggestions. This paper is supported by Youth Foundation of Naval Aeronautical and Astronautical University of China, National Nature Science Foundation of Shandong Province of China ZR2012FQ010 , National Nature Science Foundations of China 61174031, 61004002, 61102167, Aviation Science Foundation of China 20110184 and China Postdoctoral Foundation 20110490266.

\section{References}

[1] J. Huang, "Adaptive synchronization between different hyper-chaotic systems with fully uncertain parameters", Physics Letters A, vo.372, (2008), pp.4799-4804.

[2] J. H. Park, S. M. Lee and O. M. Kwon, "Adaptive synchronization of Genesio-Tesi chaotic system via a novel feedback control”, Physics Letters A, vol.371, (2007), pp.263-270.

[3] J. H. Park, "Adaptive sysnchronization of Rosser system with uncertain Parameters", Chaos, solitons and fractals, no.22, (2005), pp.1-6.

[4] X. Wu , Z. Guan and Z. Wu, "Adaptive synchronization between two different hyperchaotic systems", Nonlinear Analysis, vol.68, (2008), pp.1346-1351.

[5] M. T. Yassen, "Adaptive synchronization of Rosser and lv systems with fully uncertain patameters", Chaos,solitons and fractals, no.23, (2005), pp.1527-1536.

[6] C. C. Fuh and P. C. Tung, "Controlling chaos using differential geometric method", Phys Rev Lett, vol.75, (1995), pp.2952-5.

[7] G. Chen and X. Dong, "On feedback control of chaotic continuous-time systems", IEEE Trans Circ Syst, vol.40, (1993), pp.591-601.

[8] E. N. Sanchez, J. P. Perez, M. Martinez and G. Chen, "Chaos stabilization: an inverse optimal control approach", vol.32, (2002), pp.111-4.

[9] M. Feki, "An adaptive feedback control of linearizable chaotic systems", Chaos, Solitons \& Fractals, vol.15, (2003), pp.883-90.

[10] Y. Wang, Z. Guan and X. Wen, "Adaptive synchronization for Chen chaotic system with fully unknown parameters", Chaos, Solitons \&Fractals, vol.19, (2004), pp.899-903.

[11] G. Chen and X. Dong, "From chaos to order: methodologies, perspectives and applications", Singapore: World Scientific, (1998).

[12] E. N. Lorenz, "Deterministic non-periods flows", J Atmos Sci, vol.20, (1963), pp.130-41.

[13] G. R. Chen and T. Ueta, "Yet another chaotic attractor", Int J Bifurc Chaos, vol.9, (1999), pp.1465-6.

[14] J. H. Lv and G. R. Chen, "A new chaotic attractor coined", Int J Bifurc Chaos, vol.12, no.3, (2002), pp.659-61.

[15] A. Van-ec-ek, S. C-elikovesky, "Control systems: from linear analysis to synthesis of chaos", London: Prentice-Hall, (1996).

[16] X. Wu and J. Lu, "Parameter identification and backstepping control of uncertain Lv system", Chaos, Solitons \& Fractals, vol.18, (2003), pp.721-9.

[17] Y. Yu and S. Zhang, "Adaptive backstepping control of the uncertain Lvu system", Chin Phys, vol.11, (2002), pp.1249-305.

[18] C. Wang and S. Ge, "Adaptive backstepping control of uncertain Lorenz system", Int J Bifurc Chaos, vol.11, (2001), pp.1115-9.

[19] E. M. Elabbasy, H. N. Agiza and M. M. El-Dessoky, "Adaptive synchronization of a hyperchaotic system with uncertain parameter", Chaos, Solitons and Fractals, vol.30, (2006), pp.1133-1142.

[20] F. Tang and L. Wang, "An adaptive active control for the modified Chua's circuit, Physics Letters A, vol.346, (2005), pp.342-346.

[21] M. Hu, Z. Xu, R. Zhang and A. Hu, "Parameters identification and adaptive full state hybrid projective synchronization of chaotic (hyper-chaotic) systems", Physics Letters A, vol.361, (2007), pp.231-237.

[22] Z.-M. Ge and C.-H. Yang, "Pragmatical generalized synchronization of chaotic systems with uncertain parameters by adaptive control”, Physica D, vol.231, (2007), pp.87-89.

[23] T. Gao, Z. Chen, Z. Yuan and D. Yu, "Adaptive synchronization of a new hyperchaotic system with uncertain parameters", Chaos, Solitons and Fractals vol.33, (2007), pp.922-928. 


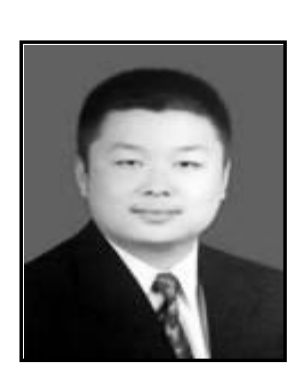

\section{Authors}

Zhanlei Shang (1974-), he was born in Yuzhou city of Henan province of China. He received his bachelor's degree in 1996 from Henan Normal University and his major is physical education and received his major's degree in 2000 from Huazhong University of Science and Technology and his major is computer technology. He is an Associate Professor and a Director of Computer Center of Zhengzhou University of Light Industry. His current research interests is database and network. He published more than 20 academic papers, and 5 of them were retrieved by EI. About 10 computer textbooks were issued under his general editorship, 3 of them were respectively awarded the Eleventh Five-Year Plan and the Twelfth Five-Year Plan for National Teaching Materials. In addition, He had taken charge of and participated in 8 scientific projects and 6 education reform projects.

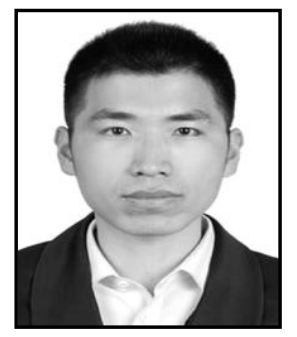

Ruiqi Wang (1980-), he was born in Xinxiang City, Henan province of China. He received his Doctor degree in Guidance, Navigation and Control in 2011 from Naval Aeronautical and Astronautical University, Yantai of China. He graduated from Naval Aeronautical and Astronautical University, Yantai of China in 2001 and received his bachelor's degree with the major of missile control and test. After that he continued his study in this school and received his master's degree and doctor's degree in 2006 and 2011 respectively. He has published more than 20 papers, where 7 papers was indexed by Ei. His present interests are control theory, missile control and bilateral control.

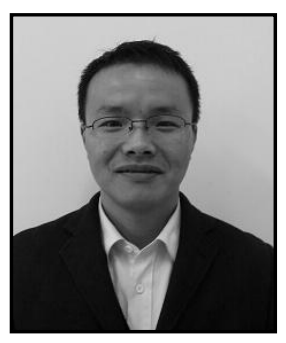

Junwei Lei (1981-), he was born in Chibi of Hubei province of China and received his Doctor degree in Guidance, Navigation and Control in 2010 from Naval Aeronautical and Astronautical University, Yantai of China. Her present interests are control theory, chaotic system control, aircraft control and adaptive control.

He was promoted to be a lecture of NAAU in 2010. His typical book named Nussbaum gain control technology of supersonic missiles was published in 2013 in China. 